\title{
ENTEROSORPTION COMBINED WITH GRANULOCYTE COLONY STIMULATING FACTOR DECREASES MELPHALAN GONADAL TOXICITY
}

\author{
O.O. Shevchuk, ${ }^{1 *}$,Ya.Ya. Bodnar ${ }^{1}$, K.I. Bardakhivska ${ }^{2}$, T.V. Datsko', A.S. Volska ${ }^{1}$, K.A. Posokhova ${ }^{1}$, \\ V.F. Chekhun', V.G. Nikolaev ${ }^{2}$ \\ ${ }^{1}$ I. Horbachevsky Ternopil State Medical University, Ternopil 46001, Ukraine \\ ${ }^{2}$ R.Y. Kavetsky Institute of Experimental Pathology, Oncology and Radiobiology, NAS of Ukraine, \\ Kyiv 03022, Ukraine
}

\begin{abstract}
Today due to improvements in cancer treatment there is an increasing number of long-term cancer survivors, many of whom suffer from infertility caused by malignancy itself and chemo- or radiotherapy. Also, anticancer therapy may cause myelosuppression. Presently granulocyte colony stimulating factor (G-CSF) is used for prevention and treatment of myelosuppression. Another treatment option used to decrease intoxication and ameliorate side effects of cancer therapy is sorption technology. The aim of our investigation was to study the efficiency of combined use of enterosorption and G-CSF to decrease gonadal toxicity of chemotherapy. Materials and Methods: Melphalan (L-PAM) injected i.v. at a single dose of $4 \mathrm{mg} / \mathrm{kg}$ to white inbred rats was used as gonadotoxic and myelosuppressing agent. Carbon enterosorbent $\mathrm{C} 2$ was administered by intragastric route as a suspension in saline at a dose of $5 \mathrm{ml}$ per $1 \mathrm{~kg}$ of rats' body weight (or $900 \mathrm{mg} / \mathrm{kg}$ of the dry mass of enterosorbent) daily for 3 days before and for 7 days after L-PAM injection. G-CSF was injected once a day for 4 days starting from the next day after L-PAM administration at a dose of $50 \mu \mathrm{g} / \mathrm{kg}$. Histological preparations of testicular tissues were examined by light microscopy. Results: Our findings have shown that melphalan caused marked damage of testicular tissues and seminiferous, especially spermatogenic epithelium. The most expressed protection of the histological structure of testes was observed when enterosorbent and G-CSF were used in combination. Conclusion: Gonadal toxicity of chemotherapy could be efficiently decreased by the combined use of enterosorption and G-CSF.
\end{abstract}

Key Words: melphalan, gonadotoxicity, carbon enterosorbent, granulocyte colony stimulating factor, histology.

In last decades, the modern methods of cancer treatment, the newest anti-neoplastic drugs and early detection of malignancy have significantly increased the number of long-term cancer survivors worldwide [1-3]. Up to 8 out of 10 children and adolescents live longer than 5 years after the cancer diagnosis [4]. Most of them experience a range of negative outcomes, in particular, infertility problem.

Gonadal toxicity in cancer patients could result from tumor growth, or be a consequence of hormonal and metabolic alterations, or chemo- and radiotherapy. The approved methods to preserve fertility in cancer patients prior to treatment include semen/oocytes cryopreservation and testicular/ovarian ultra-fast freezing following with in vitro fertilization or intracytoplasmic sperm injection to achieve the result [5-7]. For most females oocyte banking is feasible even prior to puberty onset. Males can use sperm banking only after 13-14 years of age, when spermatogenesis has started. Prepubertal testicles are more vulnerable to cytotoxic effects of chemotherapy than the adult testes [3]. For males with cancer even sperm banking could not guarantee the future fatherhood. Nearly $70 \%$ of samples taken before treatment already contain abnormalities of spermatozoa, while nearly $12-15 \%$ of patients suffer from azoospermia at the tieme of diagnosis, which makes cryopreservation impossible [3]. In these situations, there are some experimental methods to preserve

Submitted: August 9, 2016.

*Correspondence: Phone +380979006522;

E-mail: shevchukoo@tdmu.edu.ua

Abbreviation used: G-CSF - granulocyte colony stimulating factor. fertility: spermatogonial stem cell transplantation, testicular allografting and harvesting of germinal cells [3, 8]. These methods, however, are not yet approved for humans and carry some technical and ethical problems. Thus, there is still a need for effective methods to preserve fertility in long-term cancer survivors.

Cancer treatment methods such as whole-body irradiation, the use of cytostatics, especially alkylating agents, and surgical removal of reproductive organs have the highest negative impact on spermatogenetic potential and ovarian follicular function. The risks increase with younger age at the time of treatment, higher cumulative dose and the use of combinedmodality therapy $[14,15]$. Alkylating agents, especially nitrogen mustard derivates, are among anti-cancer medications that have the highest potential to cause problems with fertility, up to sterility (Table), depending on the age of patient [3].

Table. Potential to impair spermatogenesis for some chemotherapeutic medications

\begin{tabular}{lll}
\hline \multicolumn{1}{c}{ High potential } & \multicolumn{1}{c}{ Medium potential } & \multicolumn{1}{c}{ Low potential } \\
\hline Cyclophosphamide, & Cisplatin, & Vincristine, \\
ifosfamide, & carboplatin, & methotrexate dactinomycin, \\
chlormethine, & doxorubicin & bleomycin, \\
busulfan, & & mercaptopurine, \\
melphalan, & & vinblastine \\
procarbazine, & & \\
dacarbazine, & & \\
chlorambucil & & \\
\hline
\end{tabular}

Enteral sorption therapy (enterosorption) is widely used today to decrease manifestations of systemic acute and chronic intoxications, oxidative stress in cases of renal failure, hepatic insufficiency, burn toxico- 
sis, acute enteric infections [9]. Sorption technologies are also used to decrease endogenic intoxication during treatment of malignancies [10-12]. We have previously demonstrated promising results concerning protective properties of enterosorption with the use of carbon sorbent counteracting myelosuppression, an acute dose-limiting and sometimes life-threatening complications of treatment [13]. We have found that bone marrow protection was the most effective when enterosorption was used together with granulocyte colony stimulating factor (G-CSF) [13]. The aim of this investigation was to study the possibilities of such combination to preserve spermatogenesis during chemotherapy with an alkylating agent.

\section{MATERIALS AND METHODS}

The study was carried out on 40 white inbred rats weighting $200 \pm 20 \mathrm{~g}$ housed in the vivarium of R.E. Kavetsky Institute of Experimental Pathology, Oncology and Radiobiology (IEPOR). The use and care of the experimental animals have been performed in accordance with the standard international rules of biologic ethics and were approved by Institutional Animal Care and Use Committee. To examine and study gonadal toxicity we used alkylating agent melphalan (L-PAM, Alkeran, GlaxoSmithKline, Great Britain). Animals were randomly distributed into 4 groups $(n=10)$ : 1 ) intact rats; 2) rats treated with L-PAM; 3) rats treated with both L-PAM and carbon enterosorbent C2 (L-PAM + $C 2)$; 4) rats treated with L-PAM, C2 and G-CSF filgrastim (L-PAM + C2 + filgrastim).

L-PAM was injected single-dose in the tail vein at a dose of $4.0 \mathrm{mg} / \mathrm{kg}$. Filgrastim (Neupogen, Hoffman-La Roche Ltd., Switzerland) is a G-CSF analogue produced by recombinant DNA technology and used to stimulate proliferation and differentiation of granulocytes. It was injected once a day starting from the next day after L-PAM administration for 4 days at a dose of $50 \mu \mathrm{g} / \mathrm{kg}$.

Carbon granulated enterosorbent C2, produced by IEPOR, has the following parameters: bulk density of $Y=0.18 \mathrm{~g} / \mathrm{cm}^{3}$, granule diameter of 0.15 to $0.25 \mathrm{~mm}$, BET pore (Brunauer - Emmett - Teller model of pore distribution calculation) surface $2162 \mathrm{~m}^{2} / \mathrm{g}$. The administered dose of $\mathrm{C} 2$ was $5 \mathrm{ml}$ per $1 \mathrm{~kg}$ of rat's body weight (or $900 \mathrm{mg} / \mathrm{kg}$ of the dry mass of enterosorbent). A suspension of sorbent in appropriate volume of distilled water was introduced via the tube into rat stomach once a day for 3 days before the day of L-PAM injection and for 7 days after injection. Rats of L-PAMand intact groups were given equivalent volume of distilled water. Animals of intact group instead of L-PAM received intravenously equal volume of saline.

On the $8^{\text {th }}$ day after L-PAM injection, the rats were weight and sacrificed using ketamine hydrochloride general anesthesia. Testicular tissues were taken for histological analysis. Tissues were fixed in formalin, paraffin embedded and sectioned according to the standard technique. Serial $5 \mu \mathrm{m}$ sections were stained with hematoxylin and eosin and examined by light mi- croscopy using Genetic Pro Bino microscope (Delta Optical). Representative areas of the samples were microphotographed using $\times 10$ and $\times 20$ objectives using SCMOS Digital Camera and ToupView software with different magnification.

\section{RESULTS AND DISCUSSION}

Histological structure of the testis of an intact rat is presented in Fig. 1. All tubules have round shape and mostly 3-5 layers of germinal (seminiferous epithelium), which contains spermatogenic and Sertoli cells. Primary and secondary spermatocytes are located near basal membranes with darker nuclei. Spermatids and mature spermatozoa can be observed inside the lumen in appropriate quantities.

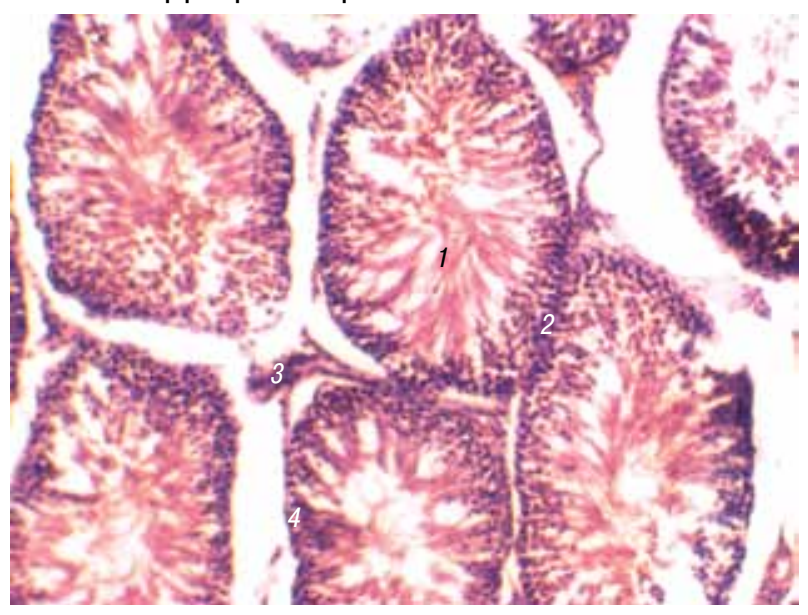

Fig. 1. Histological structure of intact rat testis. H\&E, $\times 200(1-$ mature spermatozoa; 2 - seminiferous epithelium; 3 - interstitial Leydig cell; 4 - sertoli cells)

In histological samples of the animals treated with L-PAM (Fig. 2, a) were observed the following structural changes in comparison to the intact group: deformation of the seminiferous tubules and marked enlargement of seminiferous tubules lumens; reduction and disorganization in the spermatogenic layers of epithelium (1), accompanied with sharp reduction in the number of luminal spermatozoa (2); empty lumens (3). Simultaneously, in seminiferous tubules which still preserved the large number of mature spermatozoa, there were observed detachment and damage of primary and secondary spermatocytes and spermatids (4) (Fig. 2, b).

In rats treated with both enterosorbent $\mathrm{C} 2$ and melphalan, some restoration of seminiferous epithelium and its layers could be observed, however, it did not occur in all seminiferous tubules (Fig. 3). Also, the increased number of luminal spermatids and spermatozoa was observed.

Maximal improvement of histological structures of L-PAM-affected testis was seen when enterosorbent C2 and G-CSF were administered simultaneously. Repair of epithelial layers was detected in almost all of seminiferous tubules (Fig.4).

Gonadotoxicity of melphalan is caused by both direct and indirect mechanisms. Active metabolites of the medication form cross-links with DNA and inhibit its synthesis and function, which leads to apoptotic 
cell death [16]. Melphalan also causes direct partial destruction of gonadal tissues [17]. Like all alkylating agents, it has radiomimetic properties and causes intensive oxidative stress, forming reactive oxygen species which cause indirect damage of seminiferous epithelium [18].
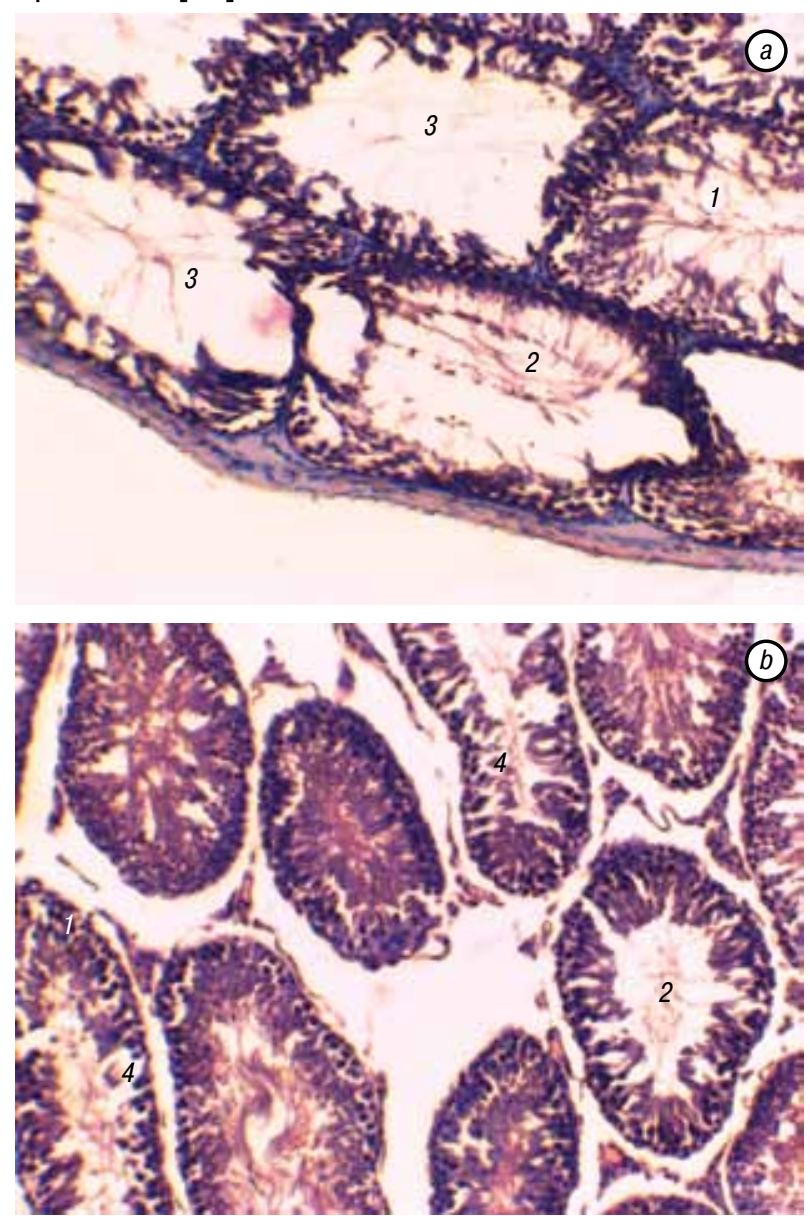

Fig. 2. Histological structure of testis of L-PAM treated rats. $\mathrm{H} \& \mathrm{E}, \times 100$. Description is in the text

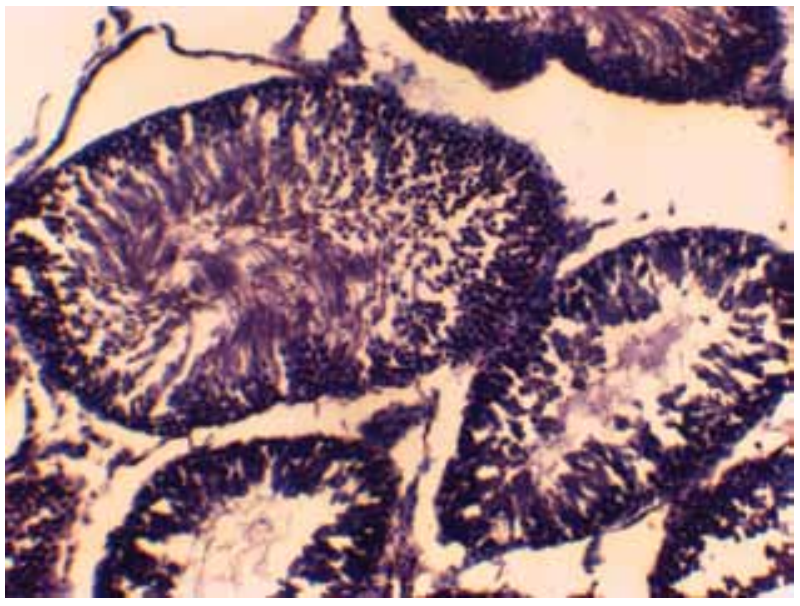

Fig. 3. Histological structure of testis of a rat from L-PAM + C2 - treated group. H\&E, $\times 100$

In conclusion, melphalan at a dosage of $4 \mathrm{mg} / \mathrm{kg}$ causes marked changes in histologic structures of rat testes, including deformation of seminiferous epithelium, reduction and disorganization of spermatogenic epithelium with its maturing forms and spermatozoa. Enterosorption with carbon enterosorbent
C2 decreases the damage of testicular tissues and increases the number of spermatocytes, spermatids and spermatozoa in the large part, but not all seminiferous tubules. Maximal positive histological improvements of the testicular structures are observed when enterosorption and G-CSF are administered together.
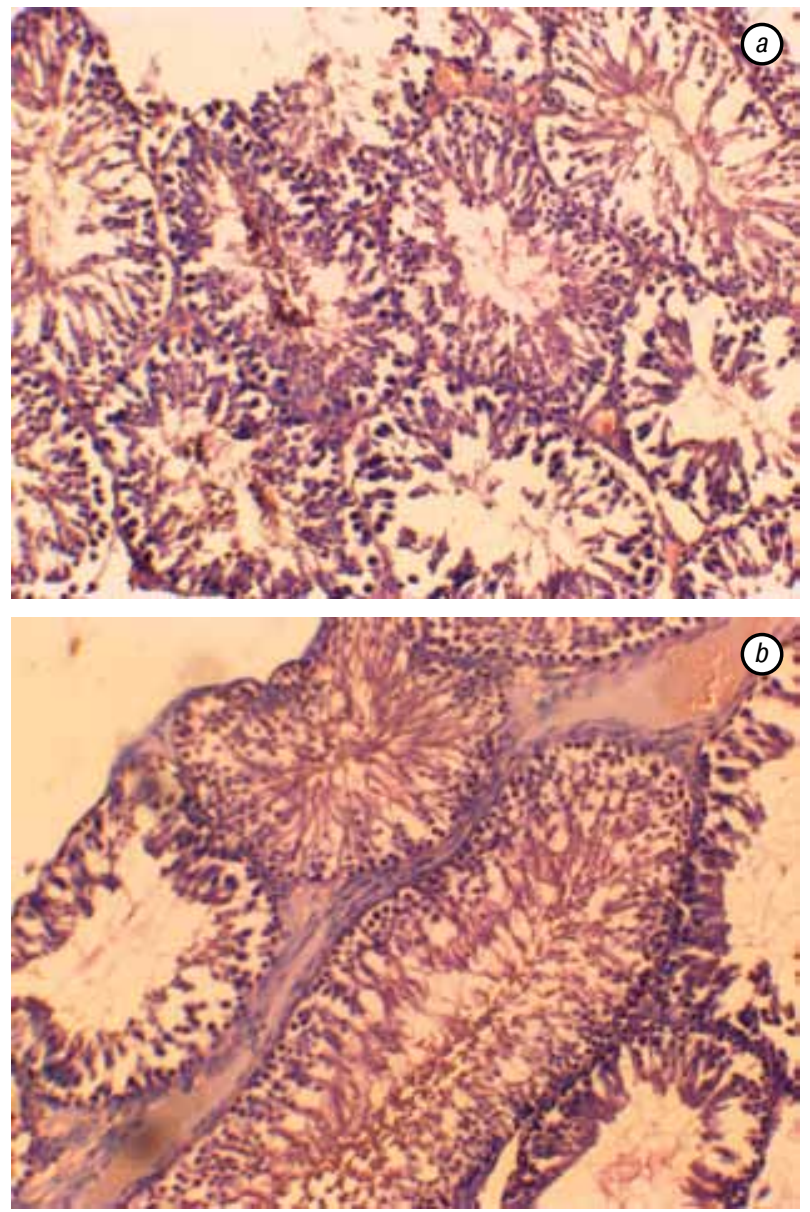

Fig. 4. Histological structure of testis of rats from L-PAM + C2 + filgrastim - treated group. H\&E, $\times 100$

Preservation of fertility in cancer patients becomes an important issue because of steadily increasing number of cancer survivors. The positive effects of combined use of carbon enterosorbent and G-CSF reported in this study point to the need for further functional investigation and subsequent implementation of these results into clinical practice.

\section{REFERENCES}

1. Stensvold E, Magelssen H, Oskam IC. Fertility-preserving measures for girls and young women with cancer. Tidsskr Nor Laegeforen 2011; 131: 1429-32.

2. De Moor JS, Mariotto AB, Parry C, et al. Cancer survivors in the united states: Prevalence across the survivorship trajectory and implications for care. Cancer Epidemiol Biomarkers Prev 2013; 22: 561-70.

3. Dohle GR. Male infertility in cancer patients: Review of the literature. Int J Urol 2010; 17: 327-31.

4. Robison LL, Hudson MM. Survivors of childhood and adolescent cancer: life-long risks and responsibilities. Nat Rev Cancer 2014; 14: 61-70.

5. Geens M, Goossens E, De Block G, et al. Autologous spermatogonial stem cell transplantation in man: current obstacles for a future clinical application. Hum Reprod Update 2008; 14: 121-30. 
6. Keros V, Hultenby K, Borgstrom B, et al. Methods of cryopreservation of testicular tissue with viable spermatogonia in pre-pubertal boys undergoing gonadotoxic cancer treatment. Hum Reprod 2007; 22: 1384-95.

7. Stensvold E, Magelssen H, Oskam IC. Fertility-preserving measures for boys and young men with cancer. Tidsskr Nor Laegeforen 2011; 131: 1433-5.

8. Chiba K, Fujisawa M. Fertility preservation in men with cancer. Reprod Med Biol 2014; 13: 177-84.

9. Nikolaev VG, Samsonov V. Analysis of medical use of carbon adsorbents in China and additional possibilities in this field achieved in Ukraine. Artif Cells, Nanomed, Biotechnol 2014; 42: 1-5.

10. Muravskaya GV, Nikolaev VG, Sergeev VP, et al. Enterosorption in oncotherapy. Artif Cells, Blood Substitutes, Biotechnol 1991; 19: 167-74.

11. Nikolaev VG, Sakhno LA, Snezhkova EA, et al. Carbon adsorbents in oncology: Achievements and perspectives. Exp Oncol 2011; 33: 2-8.

12. Sakhno LA, Yurchenko OV, Maslenniy VN, et al. Enterosorption as a method to decrease the systemic toxicity of cisplatin. Exp Oncol 2013; 35: 45-52.
13. Shevchuk OO, Posokhova KA, Todor IN, et al. Prevention of myelosuppression by combined treatment with enterosorbent and granulocyte colony-stimulating factor. Exp Oncol 2015; 37: 135-8.

14. Meistrich ML. The Effects of chemotherapy and radiotherapy on spermatogenesis in humans. Fertil Steril 2013; 100: $1-14$.

15. Lee PA, Rogol A, Houk CP. Optimizing potential for fertility: fertility preservation considerations for the pediatric endocrinologist. Endocrinol Metab Clin North Am 2009; 38: $761-75$.

16. Bedoschi G, Navarro PA, Oktay K. Chemotherapyinduced damage to ovary: mechanisms and clinical impact. Future Oncol 2016 [Epub ahead of print].

17. Turgeman O, Medical R, Blumenfeld Z. Minimizing the doxorubicin-induced gonadotoxicity by sphingosine1-phosphate analogue FTY720. Am J Clin Exp Obstet Gynecol 2015; 2: 24-33.

18. Shevchuk OO, Posokhova KA, Sidorenko AS, et al. The influence of enterosorption on some haematological and biochemical indices of the normal rats after single injection of melphalan. Exp Oncol 2014; 36: 94-100. 